\title{
Potencial visual evocado em portadores de hanseníase
}

\author{
Visual evoked potential in leprosy patients
}

\author{
João Lins de Andrade Neto ${ }^{1}$ \\ Ana Paula Cunha ${ }^{2}$ \\ Raquel Barbosa Paes Barreto ${ }^{3}$ \\ Tiago Eug'ênio Faria de Arantes ${ }^{4}$ \\ Laura Patrícia Ferreira Santos ${ }^{5}$
}

\section{RESUMO}

Objetivo: Investigar as possíveis alterações no potencial visual evocado em portadores de hanseníase. Métodos: Foram realizados exames de potencial visual evocado em 13 portadores de hanseníase, cinco da forma multibacilar e oito da paucibacilar, no momento do diagnóstico da doença. O grupo controle foi formado por 15 indivíduos saudáveis, sem hanseníase. Resultados: Os valores das latências variaram de 102,0 a 120,5 ms, commédia 110,1 $55,7 \mathrm{~ms}$. Na forma multibacilar, os valores variaram de 109,0 a 120,0 ms, média 111,1 +5,4 ms. Na paucibacilar, de 102,0 a 120,5 ms, com média de 109,5 $56,1 \mathrm{~ms}$. Os valores das latências foram significantemente maiores nos pacientes com hanseníase $(\mathrm{p}<0,0001)$, mesmo se forem comparadas, separadamente, as formas multibacilar e paucibacilar. Não houve, porém, diferença significante quando se compararam os grupos pauci e multibacilar. Conclusão: Os valores das latências foram significantemente maiores nos pacientes com hanseníase, sendo recomendável a realização de PVE nesses pacientes, como forma de investigar precocemente suas complicações, bem como prevenir seus danos.

Descritores: Hanseníase; Potenciais evocados visuais; Electrofisiologia
${ }^{1}$ Residente da Fundação Altino Ventura - Recife (PE) Brasil.

Oftalmologista do Departamento de Uveítes da Fundação Altino Ventura e do Hospital de Olhos de Pernambuco - Recife (PE) - Brasil.

Residente da Fundação Altino Ventura - Recife (PE) Brasil.

${ }^{4}$ Residente da Fundação Altino Ventura - Recife (PE) Brasil.

${ }^{5}$ Oftalmologista do Departamento de Neuroftalmologia da Fundação Altino Ventura e do Hospital de Olhos de Pernambuco - Recife (PE) - Brasil.

Endereço para correspondência: Fundação Altino Ventura. Rua da Soledade, 170 - Recife (PE) CEP 50070-040

E-mail: fav@fundacaoaltinoventura.org.br

Recebido para publicação em11.07.2005

Versão revisada recebida em 12.12.2005

Aprovação em 16.12.2005

Nota Editorial: Depois de concluída a análise do artigo sob sigilo editorial e com a anuência da Dra. Adriana Berezovsky sobre a divulgação de seu nome como revisora, agradecemos sua participação neste processo.

\section{INTRODUÇÃO}

A hanseníase é uma doença causada pelo Mycobacterium leprae, que acomete principalmente pele, nervos periféricos e olhos ${ }^{(1)}$, constituindo-se um problema de saúde pública nos países em desenvolvimento, pelo alto índice de complicações e seqüelas irreversíveis ${ }^{(2)}$. No Brasil a taxa de prevalência no ano de 2003 foi de 4,52 por 10.000 habitantes $^{(3)}$, diferente da observada no Mercosul $(6,03)^{(4)}$.

A sua transmissão se dá pelo contato íntimo e prolongado com pacientes que eliminam bacilos pela pele ou secreções nasais ${ }^{(1)}$. A doença compreende duas formas: a multibacilar (virchowiana e dimorfa) e paucibacilar (tuberculóide e indeterminada), sendo a primeira altamente contagiante ${ }^{(2)}$.

A necessidade de se estudar essas possíveis alterações é corroborada pelo fato de se observar, ainda, alta prevalência da hanseníase no Brasil, fato este que implica em cada vez mais pacientes com deficiências que oneram o sistema previdenciário e de saúde dos brasileiros ${ }^{(2)}$.

O potencial visual evocado (PVE), é um exame onde se verifica a integridade das vias ópticas, bem como a condução do estímulo nervoso pela mesma, constituindo-se em um exame não invasivo e objetivo, bastante utilizado pelos neurologistas e neuroftalmologistas ${ }^{(5)}$.

Tem sido observado, em estudos recentes, que ocorre uma lentificação no padrão de resposta do PVE em portadores de hanseníase, comparado a indivíduos sãos ${ }^{(6-7)}$. Isto pode contribuir com a hipótese de que os nervos centrais também são acometidos nesta doença ${ }^{(5,8)}$. 
O objetivo do estudo é verificar as possíveis alterações no PVE em portadores de hanseníase.

\section{MÉTODOS}

O estudo prospectivo foi realizado na Fundação Altino Ventura, no período de agosto a outubro de 2004.

Foram realizados exames de PVE em 13 portadores de hanseníase, sendo cinco da forma multibacilar e 8 paucibacilar, no momento do diagnóstico, isto é, com doença ativa. O grupo controle foi formado por 15 indivíduos saudáveis, sem hanseníase, com exame oftalmológico normal.

Foram realizados potenciais visuais evocados transientes, pelo aparelho LKC EPIC-3000, monoculares, obtidos com estímulos de reversão de padrões do tipo tabuleiro de xadrez, com ângulo visual de 15 'e taxa de reversão de $1,9 \mathrm{~Hz}$ (2 reversões/ segundo). Os registros foram captados com eletrodos de cúpula de ouro, sendo o ativo posicionado em Oz (occiput médio), o eletrodo de referência em $\mathrm{FPz}$ (fronte) e o eletrodo terra posicionado em $\mathrm{Cz}$ (vértex). As respostas consideradas para análise representam uma média de, no mínimo, 100 apresentações dos estímulos.

O grupo estudo foi composto de 8 pacientes do gênero masculino e 5 do feminino. A idade variou de 15 a 51 anos, média de $37,9 \pm 6,2$. No grupo controle 7 eram do gênero masculino e 8 do feminino. A idade variou de 17 a 55 anos, média de 38,3 $\pm 5,8$.

Foram incluídos portadores de hanseníase, sem alterações neurológicas previamente averiguadas, glaucoma, miopia ou ambliopia, condições que podem alterar o resultado do exame. Foram excluídos pacientes com alterações neurológicas de qualquer espécie, os portadores de glaucoma, miopia ou ambliopia.

Nenhum paciente dos grupos estudados consumia regularmente bebida alcoólica e apenas um paciente de cada grupo era fumante. $\mathrm{O}$ estado geral de saúde dos pacientes com hanseníase era considerado bom pela equipe de clínica médica assistente.

Foram avaliados os exames de PVE em 25 olhos no grupo estudo, já que um dos pacientes possuía um olho amblíope. No grupo controle foi avaliado 30 olhos. O PVE realizado foi o de padrão reverso, nos tamanhos 16x16 e 32x32.

Os resultados foram expressos pelos valores das latências em milisegundos (ms) observadas nos exames, bem como calculadas as médias e desvios padrão. Foi utilizado o teste $\mathrm{t}$ de Student, para amostras não pareadas. Valores de $\mathrm{p}<0,05$ foram considerados significantes.

O projeto de investigação foi aprovado pelo Comitê de Ética da FAV. Os pacientes e voluntários incluídos no estudo, assinaram um termo de consentimento livre-esclarecido.

\section{RESULTADOS}

A latência, em milisegundos, do componente P100, observadas nos exames de PVE dos portadores de hanseníase, de acordo com suas formas clínicas, estão listadas na tabela 1 . Foi considerado valor normal até $100 \mathrm{~ms}$ e valores anormais acima de $100 \mathrm{~ms}$. Os valores variaram de 102,0 a $120,5 \mathrm{~ms}$, com média $110,1 \pm 5,8 \mathrm{~ms}$ e mediana de $111,5 \mathrm{~ms}$. Considerando-se apenas a forma multibacilar, os valores variaram de 102,0 a $120,0 \mathrm{~ms}$, média $111,1 \pm 5,4 \mathrm{~ms}$ e mediana de $109,5 \mathrm{~ms}$. Na forma paucibacilar, de 102,0 a 120,5 ms, com média de 109,5 $\pm 6,1 \mathrm{~ms}$ e mediana de $107,5 \mathrm{~ms}$. No grupo controle, os valores variaram de 93,0 a $110,0 \mathrm{~ms}$, média $101,9 \pm 5,3 \mathrm{~ms}$, mediana de $103,7 \mathrm{~ms}$ (Tabela 2).

A média dos valores das latências foi significantemente maior nos pacientes com hanseníase $(\mathrm{p}<0,0001)$. O mesmo evento ocorrendo quando se comparam separadamente as formas multibacilar e paucibacilar. Não houve, porém, diferença significante quando se comparou as médias das latências dos grupos pauci e multibacilar.

No geral, os valores das latências foram significantemente maiores nos pacientes com hanseníase (Figura 1).

\begin{tabular}{|c|c|c|c|}
\hline & OD & OE & Forma clínica \\
\hline 1 & 120,0 & 109,5 & Multibacilar \\
\hline 2 & - & 111,5 & Multibacilar \\
\hline 3 & 118,0 & 109,0 & Multibacilar \\
\hline 4 & 112,5 & 109,5 & Multibacilar \\
\hline 5 & 102,0 & 108,0 & Multibacilar \\
\hline 6 & 103,0 & 105,5 & Paucibacilar \\
\hline 7 & 103,0 & 108,0 & Paucibacilar \\
\hline 8 & 117,0 & 102,0 & Paucibacilar \\
\hline 9 & 115,5 & 113,0 & Paucibacilar \\
\hline 10 & 109,0 & 105,0 & Paucibacilar \\
\hline 11 & 115,0 & 104,0 & Paucibacilar \\
\hline 12 & 118,0 & 120,5 & Paucibacilar \\
\hline 13 & 107,5 & 106,0 & Paucibacilar \\
\hline
\end{tabular}

\begin{tabular}{|rrr|}
\hline \multicolumn{3}{|c|}{ Tabela 2. Valores das latências P100 (ms) dos indivíduos do grupo } \\
controle
\end{tabular}




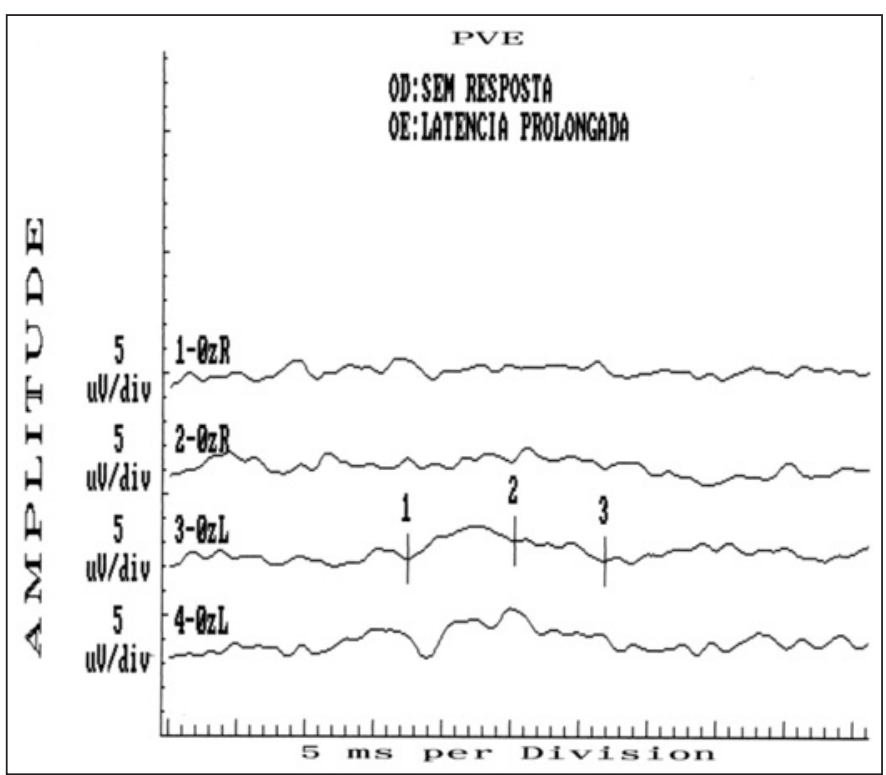

Figura 1 - Potencial visual evocado em portador de doença de Hansen: Latência prolongada no olho esquerdo. Olho direito sem resposta.

\section{DISCUSSÃO}

Devido ao fato de ser endêmica, a hanseníase no Brasil tem sido cada vez mais estudada. Políticas públicas para seu controle, aparentemente têm sido ineficazes, pois a freqüência desta doença não tem diminuído ${ }^{(5)}$. Por outro lado, as complicações oculares em portadores da doença de Hansen, na vigência de tratamento, têm sido relatadas em outros países, mesmo após completarem o tratamento com o uso de várias drogas ${ }^{(9-10)}$. O presente estudo não observou, portanto, alterações oculares importantes. Isto se deve ao fato de ser incluído pacientes da forma paucibacilar, a qual raramente acomete o olho.

O potencial visual evocado (PVE) é um exame realizado de forma rotineira em pacientes onde se suspeita de alterações nas vias ópticas, não constituindo rotina sua realização em pacientes com hanseníase, já que o conhecimento sobre a doença apenas levava em conta tão somente as alterações dos nervos periféricos ${ }^{(1-2)}$.

No presente estudo foi demonstrado que ocorre uma lentificação da condução do estímulo nervoso através da via ópti$\mathrm{ca}$, tanto na forma multibacilar, como na paucibacilar. Estudos anteriores já demonstraram essa alteração, porém apenas avaliaram apenas a forma multibacilar ${ }^{(6-7)}$. Alterações na amplitude também foram observadas, mas este estudo apenas se propôs a avaliar a latência.

As causas para estas alterações também em nervos centrais ainda não estão esclarecidas, embora a hipótese de que ocorra um fenômeno de desmielinização tanto no sistema nervoso central como em nervos periféricos possa justificar a neuropatia associada ao mencionado processo de lentificação da condução nervosa.
Torna-se necessário, portanto, uma investigação mais específica com uso de recursos histológicos e imunológicos, para se aventar teorias mais fidedignas que tragam suporte às hipóteses que justifiquem essas alterações.

A partir do conhecimento sobre estas alterações, poderá se tornar obrigatória a realização de PVE nesses pacientes, como forma de investigar as complicações na condução do estímulo nervoso na via óptica, possibilitando, eventualmente, a prevenção dos seus danos.

\section{CONCLUSÃO}

Os valores das latências foram significantemente maiores nos pacientes com hanseníase, sendo recomendável realização de PVE nesses pacientes, como forma de investigar precocemente suas complicações, bem como, eventualmente, prevenir seus danos.

\section{ABSTRACT}

Purpose: To verify the possible alterations in visual evoked potential of leprosy patients. Methods: Visual evoked potential examinations were performed in 13 Hansen disease patients, 5 of them with the multibacillary form and 8 with the paucibacillary. This was done at the moment of the diagnosis. The control group enrolled 15 health individuals, without Hansen disease. Results: The measurement result varied from 102.0 to $120.5 \mathrm{~ms}$, with a mean of $110.1 \pm 5.7 \mathrm{~ms}$. In the multibacillary form, the values varied from 109.0 to $120.0 \mathrm{~ms}$, mean $111.1 \pm 5.4$. In the paucibacillary, the values varied from 102.0 to $120.5 \mathrm{~ms}$, with a mean of $109.5 \pm 6.1 \mathrm{~ms}$. The latency values were significantly greater in Hansen disease patients $(\mathrm{p}<0.0001)$, even if multibacillary and paucibacillary forms were compared separately. However, there was no significant difference when the pauci- and multibacillary groups were compared. Conclusion: The latency values were significantly greater in leprosy patients. A recommendation can be made for these patients, which is to be submitted to early visual evoked potential as a rule in order to investigate ocular complications, as well as to prevent damages.

Keywords: Leprosy; Evoked potentials, visual; Eletrophysiology

\section{REFERÊNCIAS}

1. Kanski JJ. Oftalmologia clínica: uma abordagem sistemática. 4a ed. Rio de Janeiro: RioMed; 2004.

2. Oréfice F, Boratto LM. Caderno de uveítes. Rio de Janeiro: Sociedade Brasileira de Uveítes; 1994.

3. Anuário de informações em saúde. Brasília (DF): Ministério da Saúde; IBGE. 2003.

4. Vazquez FA, Varela NN, Antola MC, Wand-Del-Rey ML, Leguizamon OR. Hansen disease in Mercosur. Acta Leprol. 1996;10(2):79-84. Id: Spa.

5. De Oliveira CR, De Alencar Mde J, De Sena Neto AS, Lehman LF, Schreuder 
PA. Impairments and Hansen's disease control in Rondonia state, Amazon region of Brazil. Lepr Rev. 2003;74(4):337-48.

6. Dantas AM, Spielmann A, Lalanne M, Corbe C. Neuro-oftalmologia. Rio de Janeiro: Cultura Médica; 1999.

7. Ulvi H, Yigiter R, Yoldas T, Erdem I, Mungen B. Study of visual evoked potentials in the assessment of the central optic pathways in leprosy patients. Neurol Sci. 2003;24(5):346-50.
8. Kochar DK, Gupta DV, Sandeep C, Halwai M, Kumawat BL. Study of brain stem auditory-evoked potentials (BAEPs) and visual-evoked potentials (VEPs) in leprosy. Int J Lepr Other Mycobact Dis. 1997;65(2):157-65.

9. Nepal BP, Shrestha UD. Ocular findings in leprosy patients in Nepal in the era of multidrug therapy. Am J Ophthalmol. 2004;137(5):888-92.

10. Kondo S. [Ocular impairment in leprosy]. Nihon Hansenbyo Gakkai Zasshi. 2004;73(1):23-35. Review. Japanese.

\section{Congresso de Oftalmologia e 8* Congresso de Auxiliar da USP*}

\section{4 с 26 de Novembro de 2006}

\section{Centro de Convenções Rebouças Sã॰ Paul• - SP}

\section{INFORMAÇÕES: Tel.: (11) 5575-0254 - Fax: (11) 5539-2186 \\ E-mail: info@congressousp.com.br \\ Home page: www.congressousp.com.br}

*Participação neste evento conta 10 pontos para a revalidação do título de especialista em oftalmologia 